\title{
Formulation and Characterization of Chondroitin Sulfate Nanoparticle with Chitosan as Polymer and Kappa Carrageenan as Crosslinker Using the Ionic Gelation Method
}

\section{Danni Ramdhani*}

Department of Pharmaceutical Analysis and Medicinal Chemistry, Faculty of Pharmacy, Universitas Padjadjaran, Indonesia

\begin{abstract}
Chondroitin sulfate is a widely used dietary supplement formulations, effective for the treatment of osteoarthritis In oral preparations, chondroitin sulfate has low bioavailability. The alternative to improve bioavailability of a drug is by using nanoparticle technology. This study was aimed to formulate and characterize chondroitin sulfate nanoparticles with chitosan as polymer and kappa carrageenan as crosslinker. Nanoparticles were prepared by using ionic gelation method that was based on the electrostatic interaction between opposite charges. Characterization of nanoparticles were performed by measuring $\mathrm{pH}$, particle size, polydispersity index, zeta potential, and examination of infrared spectrum. The results showed that $\mathrm{F} 1$ containing $0.1 \%$ chitosan, $0.1 \%$ chondroitin sulfate, and $0.05 \%$ kappa carrageenan at volume ratio of 10:1:1 was the best formula with an average particle size $582.9 \mathrm{~nm}$, polydispersity index value of 0.324 , and zeta potential value as much as $-0.47 \mathrm{mV}$.
\end{abstract}

Keywords: Chondroitin sulfate; Chitosan; Kappa carrageenan; Nanoparticles; Ionic gelation

\section{Introduction}

Osteoarthritis (OA) is a joint disorder that often occurs in the world [1]. Therapies commonly used are the nonsteroidal antiinflammatory drugs and preparations containing chondroitin sulfate. Several clinical trials showed that chondroitin sulfate has the ability to slow the progression of osteoarthritis [2-5].

In recent years, much research that utilizes nanoparticle technology for drug delivery systems [6-9]. Various studies have also been developed, come to a focus in which pharmaceutical researchers strive to improve the effectiveness of the drug in the right amount [10]. This is because the nanoparticles has many advantages such can penetrate the space between cells that small impenetrable by particles to large size, increasing the bioavailability of drugs [11-13]. In addition, the encapsulation process can maintain the stability of the active substance from enzymatic reactions during the first pass effect [14].

Natural polymers often used in the manufacture of nanoparticles are chitosan and carrageenan. Chitosan is used very well because it has properties include safe, biodegradable, biocompatible, mucoadhesive, and hydrophilic $[15,16]$. There are studies showing that the combination of chitosan-carrageenan has a promising potential as a carrier in drug delivery systems. Carrageenan types used in these studies is kappa carrageenan [17].

The method of making nanoparticles of the most interesting is the ionic gelation method. The principle of this method is the formation of particles based on ionic gelation method.

\section{Material and Method}

\section{Materials}

Chondroitin sulfate was purchased from Bioiberica. Chitosan was purchased from Biotech Surindo. Aqua destilled was purchased from Brataco. Kappa carrageenan was purchased from PT. Quadrant. Acetic acid and potassium bromide/KBr was manufactured by Merck.

\section{Methods}

The method used in this study consists of several stages as follows:
Solubility test: Solubility testing chondroitin sulfate, chitosan, and kappa carrageenan based on the characteristics of each substance. A sample of $500 \mathrm{mg}$ dissolved in the solvent and solubility observed.

Raw materials characterization by FTIR: Infrared spectrum of a sample powder of chondroitin sulfate, chitosan, kappa carrageenan, and the combination of chondroitin sulfate/chitosan/kappa carrageenan is measured. Each sample was weighed and crushed together with $\mathrm{KBr}$ in the ratio 1:100. Then placed in a mold and compressed to form pellets. Pellets are placed in the sample container on the FTIR instrument for analysis. Measurements were made at wave number $4000 \mathrm{~cm}^{-1}$ to $400 \mathrm{~cm}^{-1}$ using FTIR spectroscopy Jasco-4200 [18]. The measurement results of the spectrum compared to the standard spectrum.

Determination of particle size of the chondroitin sulfate powder: Chondoitin sulfate was determined by Scanning Electron Microscope (SEM).

Optimization of chondroitin sulfate nanoparticles: Chitosan was dissolved in $1 \%$ acetic acid ( $\mathrm{v} / \mathrm{v})$ and made various concentrations (Table 1). Kappa carrageenan dispersed in distilled water at $60^{\circ} \mathrm{C}$ and the concentration was fixed $0.05 \%$. Chondroitin sulfate dissolved in distilled water to obtain a concentration of $0.1 \%$ chondroitin sulfate. Chondroitin sulfate solution, chitosan solution, and kappa carrageenan solution was homogenized using a vortex for 1 minute and a sonicator for 20 minutes.

Each formula has been created then measured percent

*Corresponding author: Danni Ramdhani, Department of Pharmaceutica Analysis and Medicinal Chemistry, Faculty of Pharmacy, Universitas Padjadjaran Indonesia, Tel: 62227796200; E-mail: ramdhani07@gmail.com

Received November 20, 2017; Accepted November 30, 2017; Published December 07, 2017

Citation: Ramdhani D (2017) Formulation and Characterization of Chondroitin Sulfate Nanoparticle with Chitosan as Polymer and Kappa Carrageenan as Crosslinker Using the Ionic Gelation Method. J Nanomedic Nanotechnol S8: 005. doi:10.4172/2157-7439.S8-005

Copyright: (C) 2017 Ramdhani D. This is an open-access article distributed unde the terms of the Creative Commons Attribution License, which permits unrestricted use, distribution, and reproduction in any medium, provided the original author and source are credited. 


\begin{tabular}{|c|c|c|c|}
\hline \multirow{2}{*}{$\mathbf{F}$} & \multicolumn{3}{|c|}{ Concentration in \% (w/v) } \\
\cline { 2 - 4 } & Chitosan & Chondroitin Sulfate & Kappa Carrageenan \\
\hline 1 & 0,1 & 0.1 & 0.05 \\
\hline 2 & 0.15 & 0.1 & 0.05 \\
\hline 3 & 0.2 & 0.1 & 0.05 \\
\hline 4 & 0.25 & 0.1 & 0.05 \\
\hline 5 & 0.3 & 0.1 & 0.05 \\
\hline
\end{tabular}

Table 1: Composition formula of chondroitin sulfate nanoparticles.

\begin{tabular}{|c|c|c|c|}
\hline \multirow{2}{*}{$\mathbf{F}$} & \multicolumn{3}{|c|}{ Concentration (\%) } \\
\cline { 2 - 4 } & Chitosan & Chondroitin sulfate & Kappa Carrageenan \\
\hline 1 & 0.1 & 0.1 & 0.05 \\
\hline 2 & 0.15 & 0.1 & 0.05 \\
\hline 3 & 0.2 & 0.1 & 0.05 \\
\hline
\end{tabular}

Table 2: Formula of chondroitin sulfate nanoparticles.

transmitannya using UV-Vis spectrophotometer at a wavelength of $650 \mathrm{~nm}$. Three formulas have the highest percent transmittance will have to be formulated more.

Formulation of chondroitin sulfate nanoparticles: Solution of chondroitin sulfate, chitosan, and kappa carrageenan was made with a concentration in accordance with formula chosen. Solution was homogenized using a vortex for 1 minute and then a sonicator for 20 minutes, respectively. Chitosan Chitosan solution was placed in a glass beaker on a magnetic stirrer that is rotated at a speed of 1500 $\mathrm{rpm}$. Chondroitin sulfate solution was added dropwise to the chitosan solution using a syringe, and then left to stand for 60 minutes. Kappa carrageenan solution is added to the mixture and left for 30 minutes. The third formula that was created then characterized (Table 2).

\section{Characterization of Nanoparticles}

\section{pH measurement}

$\mathrm{pH}$ measurement of chondroitin sulfate nanoparticles using a $\mathrm{pH}$ meter.

\section{Measurement of particle size and polydispersity index}

The particle size and polydispersity index of nanoparticles was measured by Particle Size Analyzer (PSA). The resulting dispersion system was taken about $3 \mathrm{~mL}$ inserted into the cuvette and analyzed by means of Particle Size Analyzer. The principle of measuring particle size were visible light scattering [19].

\section{Zeta potential measurement}

Zeta potential of the sample was measured with Laser Droppler Electrophoresis (LDE) method using a Zetasizer. Zeta potential was measured to determine the nature of the particle surface charge and determine the stability of colloidal [16].

\section{Determination of nanoparticles with FTIR}

Chondroitin sulfate nanoparticles was determined by FTIR, samples placed in a special cuvette is then measured.

\section{Results and Discussions}

\section{Solubility tests}

The test results showed that the solubility of chitosan chitosan $500 \mathrm{mg}$ dissolved in $15 \mathrm{~mL}$ of $1 \%$ acetic acid, which means that 1 part chitosan dissolved in 30 parts of acetic acid. This is in accordance with the solubility of chitosan dissolved in an organic acid at a $\mathrm{pH}$ of less than 6.5 one acetic acid [15]. Amine group in chitosan will be groups protonated $\left(-\mathrm{NH}_{3}^{+}\right)$in acid solution, so that will positively charged chitosan and soluble [13-15]. Results of testing the solubility of kappa karegenan showed that $500 \mathrm{mg}$ of kappa carrageenan dissolved in 14 $\mathrm{mL}$ of distilled water temperature $60^{\circ} \mathrm{C}$, which means that the first part of kappa carrageenan dissolved in 28 parts distilled water, this is in accordance with the solubility of kappa carrageenan are soluble in hot distilled water [12].

\section{Raw materials characterization by FTIR}

Characterization of chondroitin sulfate, chitosan, kappa carrageenan, and the combination of chondroitin sulfate/chitosan/ kappa carrageenan has been done using FTIR. This characterization includes qualitative analysis aims to determine the purity of the material based on the spectrum of the sample compared to the reference spectra (Figure 1).

Characterization of the peak of the spectrum of chondroitin sulfate sample that is at $3428.44 \mathrm{~cm}^{-1}$ indicate the presence of hydroxyl group (OH-free) and the symmetric $\mathrm{NH}_{2}$ stretching, at wave number 2931.88 $\mathrm{cm}^{-1}$ indicate the presence of a chain aliphatic C-H, at $1629.07 \mathrm{~cm}^{-1}$ showed the group $\mathrm{C}=\mathrm{O}$, at $1565.31 \mathrm{~cm}^{-1}$ (deformation $\mathrm{N}-\mathrm{H}$ ), 1244.06 $\mathrm{cm}^{-1}$ indicated the group $\mathrm{O}-\mathrm{SO}_{3}$, and the wave number $1061.84 \mathrm{~cm}^{-1}$ shows the carboxyl group $(\mathrm{C}-\mathrm{O})$. The infra-red spectrum indicated that the chondroitin sulfate sample accordance with the reference (Figure 2) [13].

Based on wave numbers of samples, chitosan showed peaks characteristic of the group $\mathrm{C}=\mathrm{O}$ stretching (amide I) at $1653.44 \mathrm{~cm}^{-1}$ and $\mathrm{NH}$ stretching (amide II) at $1596.51 \mathrm{~cm}^{-1}[14,15]$. Characterization of others can be seen at the peak of $3447.64 \mathrm{~cm}^{-1}$ that showed stretching hydroxyl group $(\mathrm{OH})$ and $\mathrm{NH}_{2}$. In addition, the chain glycosidic (-C-O-C-) which looks at wave number $1077.22 \mathrm{~cm}^{-1}$ (Figure 3) [13].

The pattern of kappa carrageenan sample in the $4000-400 \mathrm{~cm}^{-1}$ had similarities with the reference. Characterization of wave numbers of kappa carrageenan showed several peaks, one peak whose credibility is at $1253.33 \mathrm{~cm}^{-1}$ indicates that the sulfate group $(\mathrm{O}=\mathrm{S}=\mathrm{O})$, the peak of $1057.50 \mathrm{~cm}^{-1}$ showed the chain glycosidic, peaks at 926,84 $\mathrm{cm}^{-1}$ corresponding to 3,6-anhidrogalaktosa and peak $843.86 \mathrm{~cm}^{-1}$ in accordance with the galactose-4-sulfate (Figure 4) [13-15].

Typical peak contained in chondroitin sulfate, chitosan, and kappa carrageenan identified in the spectrum of this mixture. Typical peak kappa carrageenan identified as sulfate group at $1252 \mathrm{~cm}^{-1}$, galactose-4-

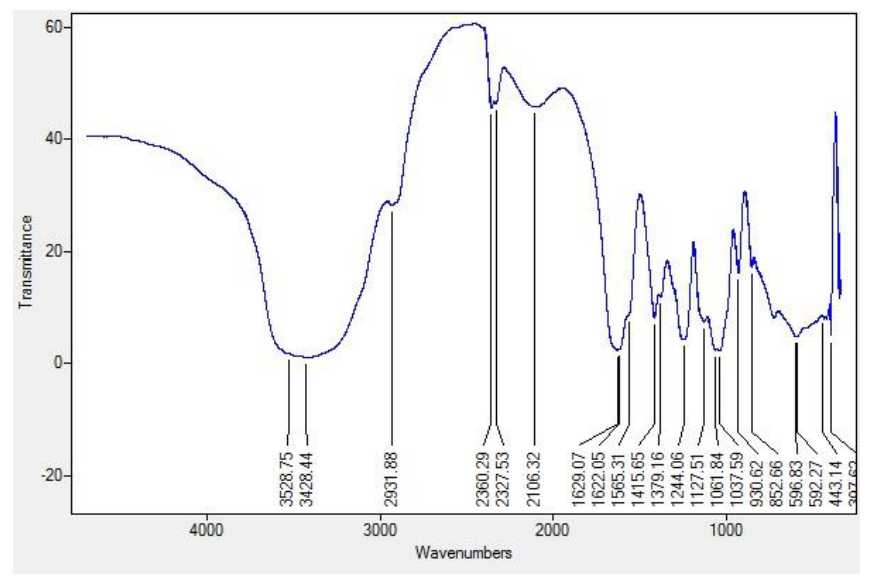

Figure 1: Spectrum of chondroitin sulfate sample. 


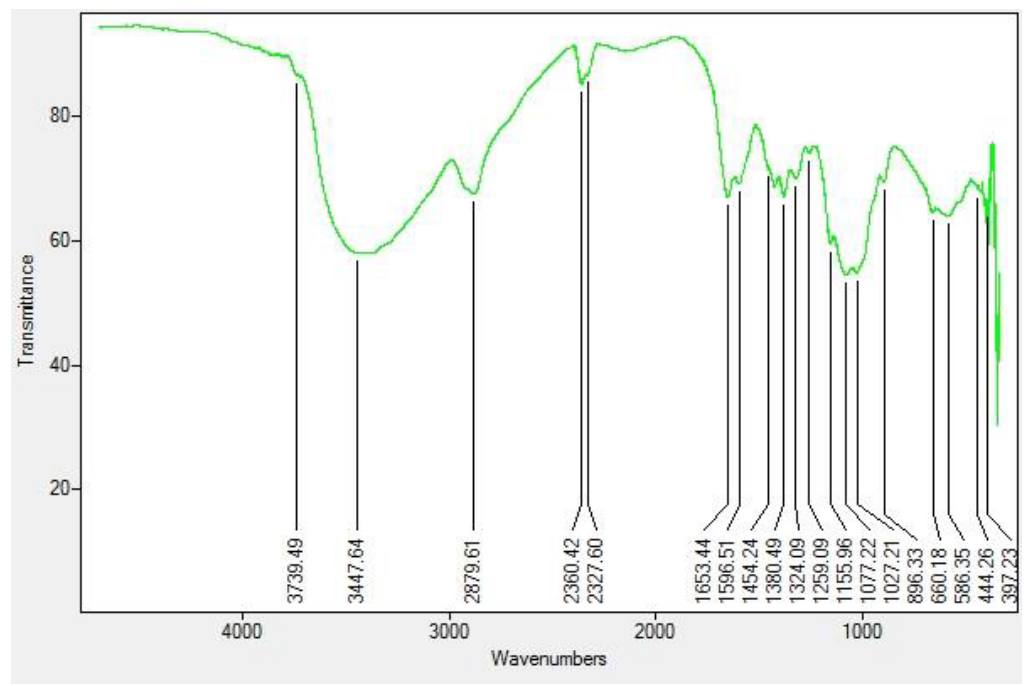

Figure 2: Spectrum of chitosan sample.

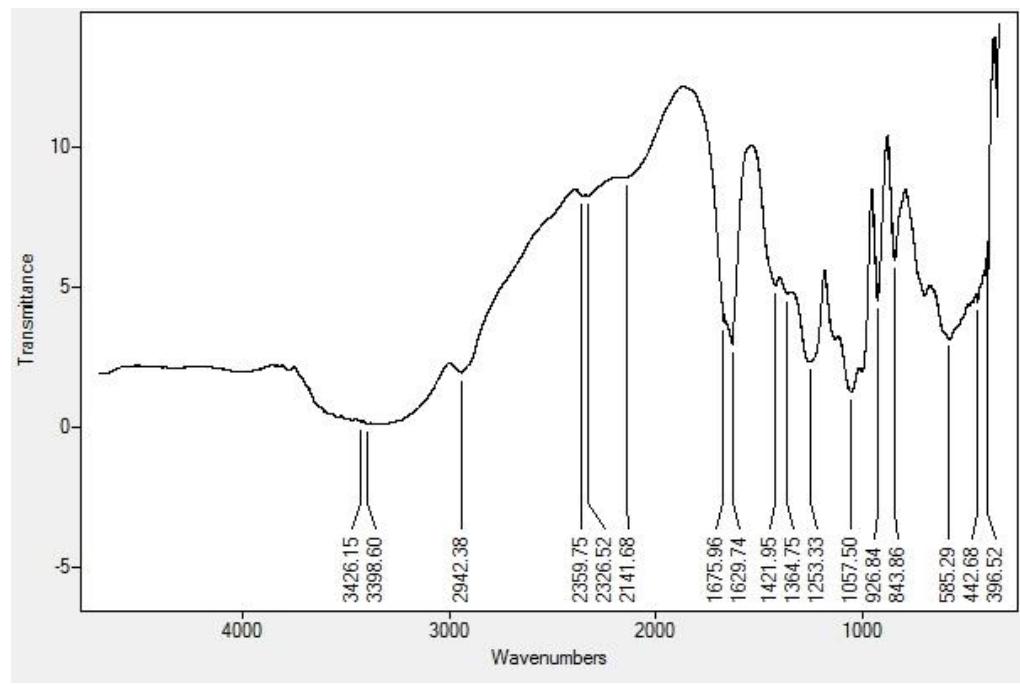

Figure 3: Spectrum of kappa carrageenan sample.

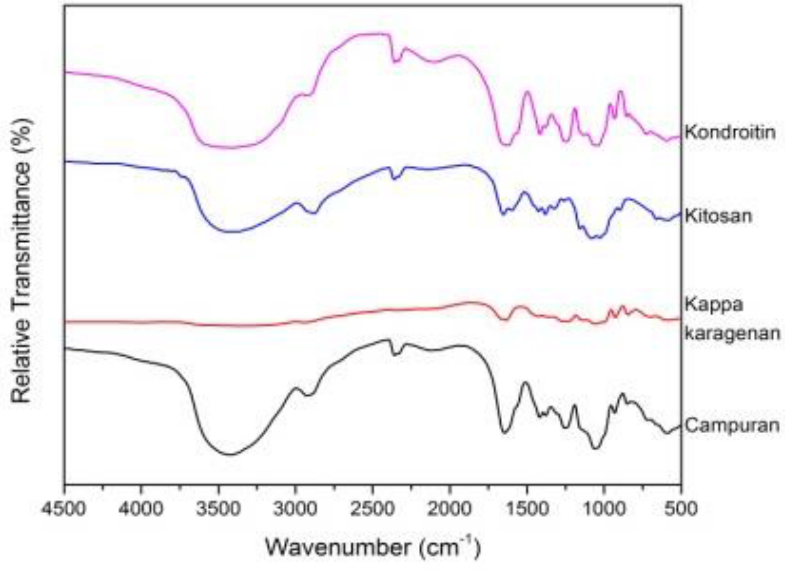

Figure 4: Spectrum of raw materials. sulfate at $849 \mathrm{~cm}^{-1}$, and 3.6-anhidrogalaktosa at $930 \mathrm{~cm}^{-1}$. This suggests that the mixture of powders chondroitin sulfate, chitosan, and kappa carrageenan did not interaction.

\section{Determination of particle size of the chondroitin sulfate powder}

Chondroitin sulfate powder was determinated by Scanning Electron Microscope (SEM) aims to determine the initial size.

Based on Figure 5 it was known that the size of chondroitin sulfate before it is formulated into nanoparticles was $1.39 \mu \mathrm{m}$ to $2.64 \mu \mathrm{m}$. The results of this determination can be compared with the size of chondroitin sulfate that had been formulated with the polymer and crosslinker into nanoparticles.

\section{Optimization of chondroitin sulfate nanoparticles}

The greater the percent transmittance indicated that the size of the particles in the dispersion system is getting smaller. 
Citation: Ramdhani D (2017) Formulation and Characterization of Chondroitin Sulfate Nanoparticle with Chitosan as Polymer and Kappa Carrageenan as Crosslinker Using the Ionic Gelation Method. J Nanomedic Nanotechnol S8: 005. doi:10.4172/2157-7439.S8-005

Percent transmittance measurements performed using UV-Vis spectrophotometry at a wavelength of $650 \mathrm{~nm}$ and used distilled water as a blank. Formula was selected based on the percent transmittance was close to $100 \%$, which means that the resulting dispersion system had a transparency similar to distilled water. This is because at a wavelength of $650 \mathrm{~nm}$, the transmittance percent distilled water had a maximum of $100 \%$ (Figure 5).

The ratio of the volume of chitosan $(\mathrm{K})$, chondroitin sulfate $(\mathrm{Ks})$, and kappa carrageenan $(\mathrm{Kk})$ used in the fifth consecutive formula is 10:1:1 in units of $\mathrm{mL}$.

The results of measurements of percent transmittance by Table 3 it can be seen that from the fifth formula is made visible that Formula 1, Formula 2 and Formula 3 has a transmittance value percent higher than the formula 4 and formula 5. Based on these data the formula that will be used in the manufacture nanoparticles and characterized further was formula 1, formula 2 and formula 3 with a volume ratio chitosan:chondroitin sulfate:kappa carrageenan is $10 \mathrm{~mL}: 1 \mathrm{~mL}: 1 \mathrm{~mL}$.

\section{Formulation of chondroitin sulfate nanoparticles}

Nanoparticles chondroitin sulfate had been made with the composition formula selected based optimizations performed.

In the process of its formation, chitosan as polymer dissolved beforehand in a solution having a $\mathrm{pH}$ of acid with the aim of changing the amine group $\left(\mathrm{NH}_{2}\right)$ to $\left(-\mathrm{NH}_{3}^{+}\right)$. The group will interact positively ionized ionic negatively charged chondroitin sulfate. Kappa carrageenan is polianion added in order to stabilize the positive charge of the ammonium groups that did not interact.

\section{Characterization of nanoparticles}

pH measurement: $\mathrm{pH}$ measurement chondroitin sulfate

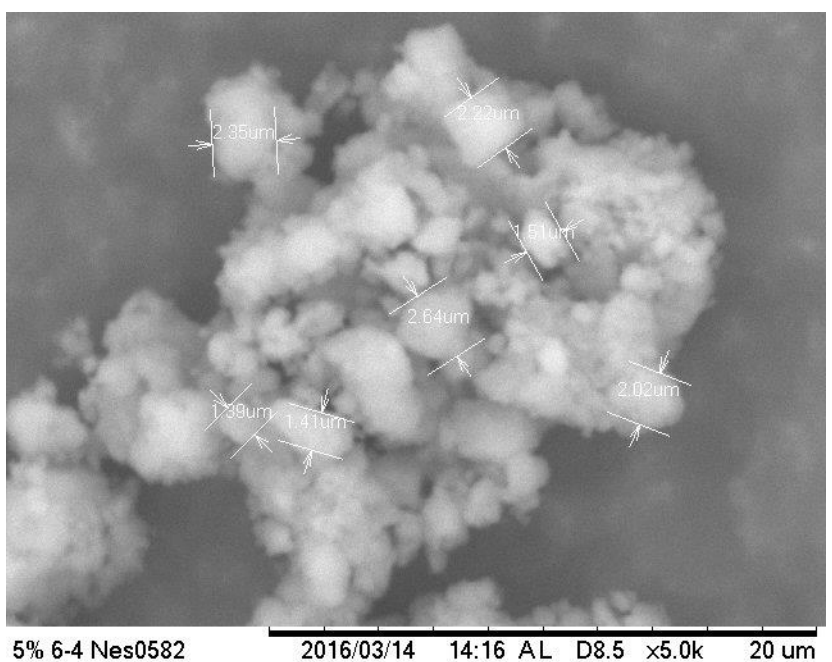

5\% 6-4 Nes0582

Figure 5: The results of the determination of particle size of the chondroitin sulfate powder.

\begin{tabular}{|c|c|c|c|c|}
\hline \multirow{2}{*}{ Formula } & \multicolumn{3}{|c|}{ Percent transmittance (\%) } & \multirow{2}{*}{ Mean } \\
\cline { 2 - 4 } & I & II & III & \\
\hline 1 & 99.28 & 99.26 & 99.26 & 99.26 \\
\hline 2 & 98.87 & 99.04 & 99.20 & 99.03 \\
\hline 3 & 99.06 & 99.04 & 99.05 & 99.05 \\
\hline 4 & 98.75 & 98.78 & 98.80 & 98.77 \\
\hline 5 & 98.37 & 98.36 & 98.35 & 98.36 \\
\hline
\end{tabular}

Table 3: Percent transmittance of chondroitin sulfate nanoparticles. nanoparticles in three formulas showed that all three had an acidic $\mathrm{pH}$. This is because the environment of the nanoparticles that was a solution of acetic acid used as a solvent chitosan. The $\mathrm{pH}$ value of the three formulas tend to increase with increasing concentrations of chitosan used.

Particle size: Determination of particle size is a primary characteristic in the manufacture of nanoparticles. The particle size was used to look at the success of the manufacture of nanoparticles, in general, the nanoparticle size range of 1-1000 nm. Particle size was determinated by Particle Size Analyzer (PSA) with the diluent water. Aside from the three formulas, particle size measurement performed also on nanocarrier that only contains the polymer and crosslinker. The aim of the nanocarrier particle size measurement was to determine the particle size of the resulting dispersion system before adding the active ingredient.

Based on the parameters of the average diameter (mean), the particle size of the F1, F2, and F3 tend to be large at more than 500 $\mathrm{nm}$. The particle size distribution can be described from three points, namely D10, D50, and D90 (Table 4).

D10 illustrates that there are $10 \%$ of the dispersion system which has a smaller particle size, while the remaining $90 \%$ has a particle size that is larger than the value stated, contrary D90 illustrates that $90 \%$ smaller particle size and $10 \%$ particle size larger. D50 or median illustrate that there are $50 \%$ of the dispersion system that has a smaller size and $50 \%$ have a particle size that is larger than the value stated. The definition when applied to the measurement results of the formula 1 which has a D10 $79.7 \mathrm{~nm}$, meaning that $10 \%$ of the particles have a particle size smaller than $79.7 \mathrm{~nm}$ and $90 \%$ of the particles have a larger particle size of $79.7 \mathrm{~nm}$.

The results of research conducted in accordance with Grenha et al. [5] which showed that the particle size of the nanocarrier chitosankappa carrageenan is in the range $350-650 \mathrm{~nm}$.

Polydispersity index: Measurement of particles using Particle Size Analyzer (PSA) may also be obtained polydispersity index value. Measurement of the value of polydispersity index aims to determine the particle size distribution which indicates the level of uniformity of size of nanoparticles produced.

Polydispersity index value measured from the third formula indicates a value below 0.5 , which means that a homogenous dispersion system formed is monodisperse. Polydispersity index value for monodisperse systems starting from 0.01 up to the value of 0.5-0.7. While the value of polydispersity index of more than 0.7 shows a very wide size distribution and may contain large particles or aggregates [14]. The smaller the value of polydispersity index showed that the narrower the particle size distribution, which means the more homogeneous particle size diameter (Table 5) [17].

Zeta potential: Zeta potential is one of the most important nanoparticle characterization. Zeta potential measurements were conducted to determine the surface charge and give an idea repulsive force among particles of the resulting nanoparticle dispersion system.

\begin{tabular}{|c|c|c|c|c|}
\hline \multirow{2}{*}{ Sample } & \multicolumn{4}{|c|}{ Particle size (nm) Mean } \\
\cline { 2 - 5 } & Mean & D10 & D50 & D90 \\
\hline F1 & 582.9 & 79.7 & 112.8 & 236.9 \\
\hline F2 & 923.5 & 134.7 & 192 & 398.9 \\
\hline F3 & 895.8 & 296.8 & 449.5 & 821.2 \\
\hline Nanocarrier & 637.2 & 96.3 & 136.7 & 284.7 \\
\hline
\end{tabular}

Table 4: Particle size of chondroitin sulfate Nanoparticles. 
The interaction between particles had an important role in the stability of the colloidal solution. In general, a dispersion system declared stable if the value of the zeta potential was more positive than $+30 \mathrm{mV}$ or more negative than $-30 \mathrm{mV}$ were determined based on electrostatic repulsion [13].

In Table 6 showed that the formula 1 containing chitosan $0.1 \%$, chondroitin sulfate $0,1 \%$, kappa carrageenan $0.05 \%$ with a volume ratio of 10:1:1 had a zeta potential value the most. But overall system of nanoparticles formed from the three formulas tend to be unstable. This is because the value of the zeta potential of the three formulas was low and did not qualify, so prone to form aggregates.

Favorably on the zeta potential like that of formula 2 and formula 3 showed that the nanoparticles produced were mostly found on the surrounding surface. This is influenced by the presence of a free amino group that can cause increased surface charge and zeta potential value generated [9].

Determination of nanoparticles with FTIR: Spectrum of Chondroitin sulfate nanoparticles was determined by Fourier Transform Infra-Red (FTIR) aims to see whether or not a shift in absorption area that appears.

Infra-red spectrum of chondroitin sulfate nanoparticles Figure 6 compared to the infra-red spectrum of chondroitin sulfate when preformulation. The results showed that the spectrum of chondroitin sulfate nanoparticles they are symmetric stretching of the hydroxyl

\begin{tabular}{|c|c|}
\hline Formula & Polydispersity index \\
\hline 1 & 0.324 \\
\hline 2 & 0.341 \\
\hline 3 & 0.297 \\
\hline
\end{tabular}

Table 5: Polidispersity index of nanoparticles.

\begin{tabular}{|c|c|}
\hline Formula & Zeta potential (mV) \\
\hline 1 & -0.47 \\
\hline 2 & +0.15 \\
\hline 3 & +0.35 \\
\hline
\end{tabular}

Table 6: Zeta potential of nanoparticles group $(\mathrm{O}-\mathrm{H})$ and $\mathrm{NH}_{2}$ which generates vibration at $3478.95 \mathrm{~cm}^{-1}$, C-H aliphatic shown at $2919.7 \mathrm{~cm}^{-1}$, and the carbonyl group is shown in $1631.48 \mathrm{~cm}^{-1}$. C-O group at $1079.94 \mathrm{~cm}^{-1}$.

Absorption area of functional groups contained in chondroitin sulfate nanoparticles experienced a slight shift compared with the absorption area chondroitin sulfate raw materials. Shifting catchment area occurs because the bonds that are formed between chondroitin sulfate with the polymer and crosslinker used. Some peaks of chondroitin sulfate that was at wavenumber $1565.31 \mathrm{~cm}^{-1}$ and 1244.06 $\mathrm{cm}^{-1}$ disappeared after being made nanoparticles, this could be caused due to the resulting dispersion system is not homogeneous. In Table 6 in mind that the value of the zeta potential of the third composition of the formula is very low, so it can be said that the resulting nanoparticles were unstable and easily aggregate.

\section{Conclusion and Suggestion}

\section{Conclusion}

The composition formula chondroitin sulfate nanoparticles can be made with various concentrations of chitosan (K) $0.1 \%, 0.15 \%$ and $0.2 \%$, while the concentration of chondroitin sulfate (Ks) and kappa carrageenan $(\mathrm{Kk})$ was fixed at $0.1 \%$ and $0.05 \%$ with a volume ratio $(\mathrm{K}: \mathrm{Ks}: \mathrm{Kk})$ is 10:1:1.

Nanoparticles best chondroitin sulfate based on the results of characterization that has been done is to use the composition of formula 1 containing chitosan $0.1 \%, 0.1 \%$ chondroitin sulfate, kappa carrageenan $0.05 \%$ with a volume ratio of $10: 1: 1$ had a $\mathrm{pH}$ value of 3.1 (acid), an average particle size (mean) $582.9 \mathrm{~nm}, 236.9 \mathrm{~nm}$ D90 value, 0.324 polydispersity index and zeta potential $-0.47 \mathrm{mV}$. Chondroitin sulfate nanoparticles formed from the formula was not stable so it has a tendency to form aggregates.

\section{Suggestion}

Future studies are recommended to complete the characterization parameters include chondroitin sulfate determination of percent adsorption, diffusion test in vitro, and morphological characterization of nanoparticles chondroitin sulfate.

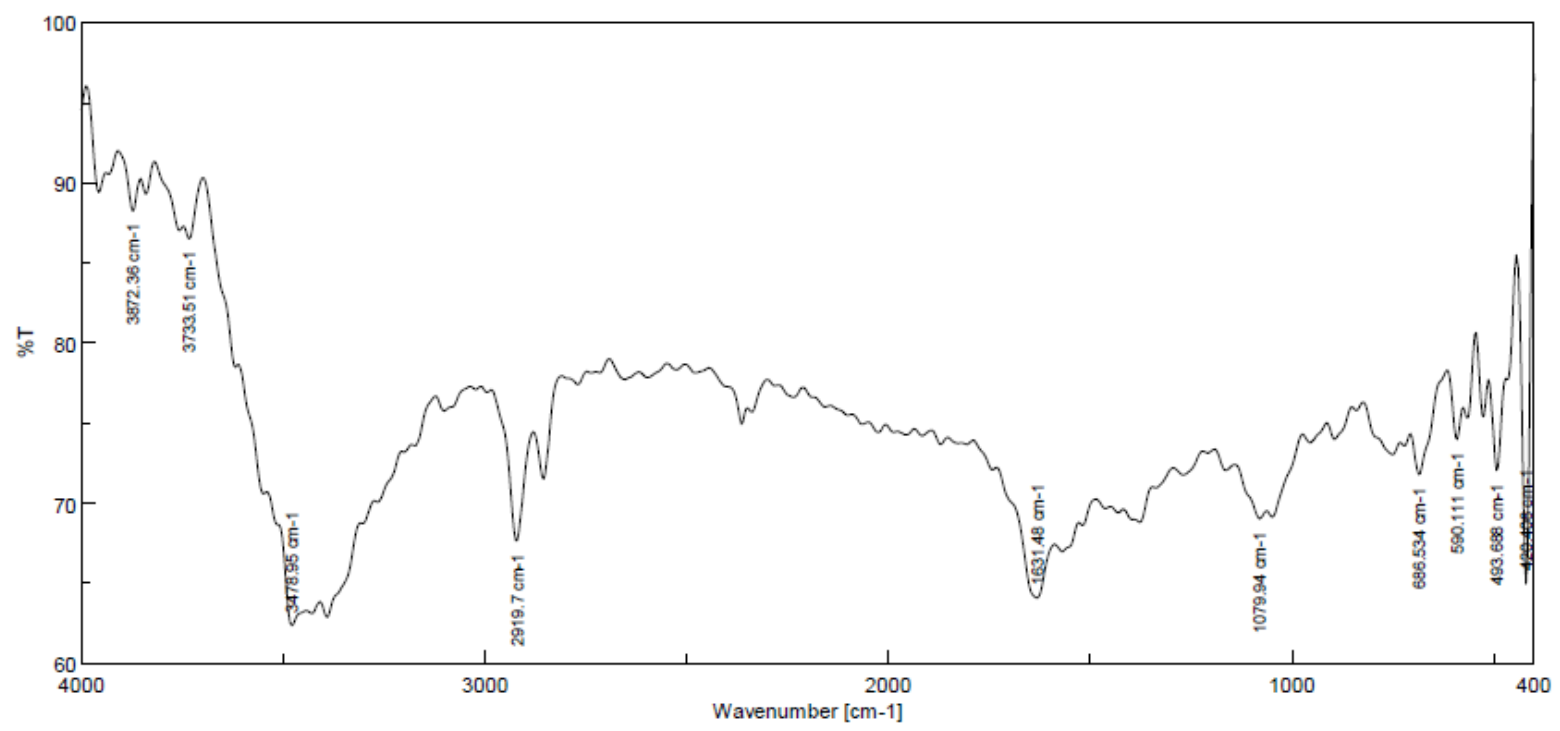

Figure 6: FTIR spectrum of chondroitin sulfate nanoparticles. 
Citation: Ramdhani D (2017) Formulation and Characterization of Chondroitin Sulfate Nanoparticle with Chitosan as Polymer and Kappa Carrageenan as Crosslinker Using the Ionic Gelation Method. J Nanomedic Nanotechnol S8: 005. doi:10.4172/2157-7439.S8-005

\section{Acknowledgments}

The author would like to thank Richie A.I. Chandra, M.S.c., Apt and Aneu Nur Utami for its cooperation in this study.

\section{References}

1. Agnihotri SA, Mallikarjuna NN, Aminabhavi TM (2004) Recent advances on chitosan-based micro and nanoparticles in drug delivery. J Controlled Release 100: 5-28.

2. Bhumkar DR, Pokharkar VB (2006) Studies on effect of pH on Cross-linking of Chitosan with Sodium Tripolyphosphate: A Technical Note. AAPS Pharm Sci Tech 7: E1-E6.

3. Buranachai T, Praphairaksit N, Muangsin N (2010) Chitosan/polyethylene glycol beads crosslinked with tripolyphoshate and glutaraldehyde for gastrointestinal drug delivery. AAPS PharmSciTech 11: 1128-1137.

4. Buzea C, Ivan IPB, Kevin R (2007) Nanomaterial and nanoparticle: Sources and toxicity. Biointerphasess 2: 170-172.

5. Grenha A, Manuela EG, Màrcia R, Vitor ES, João FM, et al. (2009) Development of new chitosan/carrageenan nanoparticles for drug delivery applications. Journal of Biomedical Materials Research 1265-1272.

6. Horiba Instruments (2014) A Guidebook to Particle Size Analysis.

7. Kumirska J, Cherwicka M, Kaczynski Z, Bychowska A, Brzozowski K, et al. (2010) Application of spectroscopic methods for structural analysis of chitin and chitosan. Mar Drugs 8: 1576-1636.

8. Lawrie G, Keen I, Drew B, Chandler-Temple A, Rintoul L, et al. (2007) Interactions between alginate and chitosan biopolymers characterized using FTIR and XPS. Bio-macromolecules 8: 2533-2541.
9. Li J, Qingrong Y (2012) Rheological properties of chitosan-tripolyphosphate complex: From suspensions to microgels. Carbohyd Polym 87: 1670-1677.

10. Li XY, Kong XY, Shi S, Zheng XL, Guo G, et al. (2008) Preparation of alginate coated chitosan microparticle for vaccine delivery. BMC Biotechnology 8: 89

11. Lockman PR, Mumper RJ, Khan MA, Allen DD (2002) Nanoparticle Technology for Drug Delivery Across the Blood-Brain Barrier. Drug Development and Industrial Pharmacy 28: 1-13.

12. Lockwood B (2007) Nutraceuticals (2ndedn), London: Pharmaceutical Press.

13. Michel BA, Stucki G, Frey D (2005) Chondroitins 4 and 6 sulfate in osteoarthritis of the knee: a randomized, controlled trial. Arthritis \& Rheumatism 52: 779-786.

14. Murdock RC, Braydich-Stolle L, Schrand AM, Schlager JJ, Hussain SM (2008) Characterization of nanoparticle dispersion in solution prior to in vitro exposure using dynamic light scattering technique. Toxicol Sci 101: 239-253.

15. Ramya R, Sudha PN, Mahalakshmi J (2012) Preparation and Characterization of Chitosan Binary Blend. IJSRP 2: 1-9.

16. Rowe RC, Sheskey PJ, Quinn ME (2009) Handbook of pharmaceutical excipients (6thedn), London: The Pharmaceutical Press 45-58.

17. The Departement of Health of UK (2013) British Pharmacopoeia 2013. London: Crown.

18. Tiyaboonchai W (2003) Chitosan nanoparticles: A promising system for drug delivery. Naresuan University Journal 11: 51-66.

19. Zhao L, Burguera EF, Xu HHK, Amin N, Ryou H, et al. (2010) Fatigue and human umbilical cord stem cell seeding characteristics of calcium phosphatechitosan-biodegradable fiber scaffolds. Biomaterials.31: 840-847. 